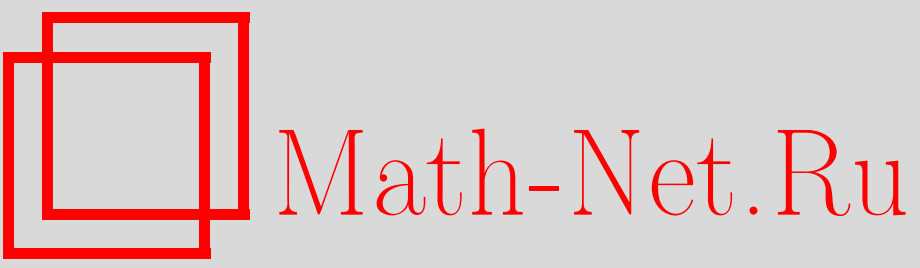

М. А. Пудовкина, Г. И. Хоруженко, Атака на шифрсистему ГОСТ 28147-89 с 12 связанными ключами, Матем. вопр. криптогр., 2013, том 4, выпуск 2, 127152

DOI: https://doi.org/10.4213/mvk88

Использование Общероссийского математического портала Math-Net.Ru подразумевает, что вы прочитали и согласны с пользовательским соглашением

http://www.mathnet.ru/rus/agreement

Параметры загрузки:

IP: 54.162 .85 .209

26 апреля 2023 г., 13:00:12 
УДК: 519.719 .2

\title{
Атака на шифрсистему ГОСТ 28147-89 с 12 связанными ключами
}

\author{
М. А. Пудовкина, Г. И. Хоруженко \\ Национальный исследовательский ядерный университет (МИФИ), Москва
}

Получено 18.IX.2012

Приведена атака на блочную шифрсистему ГОСТ 28147-89 с 12 связанными ключами. Она основана на комбинации методов бумеранга и связанных ключей и для произвольного $s$-бокса позволяет найти все 256 бит ключа шифрования. В ряде случаев ключ шифрования может быть найден на ЭВМ за приемлемое время.

Ключевые слова: ГОСТ 28147-89, метод связанных ключей, метод бумеранга

An attack on the GOST 28147-89 block cipher with 12 related keys

\section{A. Pudovkina, G. I. Khoruzenko \\ National Nuclear Research University, Moscow}

Abstract. A key-recovery attack on the full GOST 28147-89 block cipher with 12 related keys is described. The attack is based on the related-key and boomerang techniques and allows to find all 256 bits of the master key for arbitrary $s$-boxes. In some cases the attack has practical complexity.

Key words: GOST 28147-89, related-key attack, boomerang attack

Citation: Mathematical Aspects of Cryptography, 2013, vol. 4, no. 2, pp. 127-152 (Russian). 


\section{1. Введение}

Блочная шифрсистема ГОСТ 28147-89 используется в государственных и ряде коммерческих организаций Российской Федерации. Алгоритм блочного шифрования ГОСТ 28147-89 основан на схеме Фейстеля. Число раундов равно 32, размер блока текста - 64 бита, длина ключа шифрования - 256 бит. Раундовая функция алгоритма шифрования представляет собой композицию преобразований: сложения с раундовым ключом по модулю $2^{32}$, нелинейного преобразования (слой $s$-боксов) и преобразования циклического сдвига. В алгоритме развертывания ключа используются подблоки ключа шифрования в качестве раундовых ключей в определенном порядке.

Шифрсистема ГОСТ 28147-89 исследовалась с помощью разных подходов (см., например, [1] - [14]). Так в работах [1] - [3] приведены сдвиговые атаки, основанные на слабостях алгоритма развертывания ключа и раундовой функции. В работах [4] и [5] предложены атаки на основе метода отражений, а в работах [6] - [9] - атаки на основе алгебраического и разностного методов. К шифрсистеме ГОСТ 28147-89 применялась также атака на основе метода встречи посередине [10].

В последние годы появилось немало работ ( [11]- [16]), посвященных криптоанализу шифрсистемы ГОСТ 28147-89 на основе метода связанных ключей и различных обобщений разностного метода. В [11] для шифрсистемы ГОСТ 28147-89 обсуждалась возможность построения разностной характеристики для 20 и 24 раундов при наличии двух связанных ключей. Дальнейшее продвижение связано с работой [12], в которой приведена разностная характеристика, для построения которой также применены два связанных ключа. Отмечено, что в зависимости от свойств $s$-бокса на основе этой характеристики возможно атаковать редуцированную шифрсистему ГОСТ 28147-89 с 20-27 раундами. В работе [13], развивающей подход работы [12], описана атака на 31-раундовую шифрсистему ГОСТ 28147-89 на основе двух связанных ключей. Также приведен различитель, использующий два связанных ключа и отличающий алгоритм шифрования ГОСТ 28147-89 от случайной подстановки с вероятностью $1-2^{-64}$. Кроме того, предложена атака, позволяющая определить 12 бит раундового ключа 32 раунда с вероятностью 0.917 и использующая $2^{35}$ подобранных открытых текстов и $2^{35}$ шифрований.

В работе [14] приведена атака на алгоритм блочного шифрования ГОСТ 28147-89 на основе метода бумеранга со связанными ключами, которая содержит ряд ошибок и на самом деле не работает. Однако основная идея работы [14] позволяет подправить атаку и, внеся дополнительные модификации, получить работающий алгоритм. Это было сделано в работе [15]. В этой 
атаке для нахождения всего ключа шифрования при использовании $s$-боксов $\left.\widehat{s}=\left(\widetilde{s}_{8}, \ldots, \widetilde{s}_{1}\right)\right)$ из [17] требуется 18 связанных ключей и проводится $2^{26}$ зашифрований. Однако есть ряд случаев, к которым эти результаты неприменимы. Если подстановка $\widetilde{S}_{8}$ обладает линейным транслятором, то невозможно различить истинные биты ключа от ложных с помощью представленного в работе [15] различителя. Также данный различитель не позволяет найти раундовые ключи 25 и 26-го раундов. Тем самым определяются только 192 бита ключа шифрования. Для этого требуется 14 связанных ключей.

В работе [16] уменьшено число связанных ключей до двух и четырех при атаке на полнораундовую шифрсистему ГОСТ 28147-89. Она использует модифицированную усеченную характеристику работы [13]. Число связанных ключей зависит от (вероятностных) свойств $s$-бокса. Недостатком атаки является ее применимость только к некоторому множеству $s$-боксов.

В данной работе проведена модификация атаки из [15], позволяющая восстановить весь ключ шифрования с 12 связанными ключами для произвольного $s$-бокса. В ряде случаев ключ определяется на ЭВМ за практически приемлемое время . При наличии линейного транслятора для его нахождения используется модификация различителя работы [15]. Для восстановления раундовых ключей 25 и 26-го раундов применяется разностная характеристика, отличная от характеристики работы [15]. Предложенный способ нахождения ключа шифрования состоит из двух этапов. На первом этапе методом бумеранга со связанными ключами определяются множества кандидатов в раундовые ключи 32 и 31-го раундов, используя четверку связанных ключей. На втором этапе находятся множества кандидатов в раундовые ключи 30 , $29, \ldots, 25-г о$ раундов разностным методом со связанными ключами (применяются пары связанных ключей). Каждый из этапов предполагает наличие двух модификаций в зависимости от свойств подстановки $\widetilde{s}_{8}$.

\section{2. Описание шифрсистемы ГОСТ 28147-89}

Пусть $V_{n}-n$-мерное векторное пространство над полем $G F(2) ; \mathbb{N}-$ множество натуральных чисел, $\mathbb{N}_{0}=\mathbb{N} \cup\{0\} ; S(X)$ - множество всех подстановок на $X ; \oplus$ - покоординатное сложение по модулю два векторов из $V_{t}, t \in \mathbb{N} ; \alpha \lll t(\alpha \ggg t)$ - циклический сдвиг вектора $\alpha$ на $t$ бит влево (вправо); $t_{q}-$ сложение по модулю $q$; запись $x \in_{U} X$ означает, что $x$ случайно и равновероятно выбирается из множества $X ; X^{\times}=$ $=X \backslash\{0\} ; K^{[r]}-$ множество кандидатов в раундовые ключи $r$-го раунда; $\widetilde{\beta}=$ $=\left(\beta_{3}, \ldots, \beta_{0}\right) \in V_{4} ; \widehat{\beta}=\left(\widetilde{\beta}_{7}, \ldots, \widetilde{\beta}_{0}\right) \in V_{32} ; \widehat{s}=\left(\widetilde{s}_{8}, \ldots, \widetilde{s}_{1}\right), \widehat{s}: V_{32} \rightarrow V_{32}$, 


$$
\begin{gathered}
\widehat{s}(\widehat{\beta})=\left(\widetilde{s}_{8}\left(\widetilde{\beta}_{7}\right), \ldots, \widetilde{s}_{1}\left(\widetilde{\beta}_{0}\right)\right), \widetilde{s}_{i} \in S\left(V_{4}\right) ; \alpha=\left(\widehat{\alpha}^{(7)}, \ldots, \widehat{\alpha}^{(0)}\right) \in V_{256} ; \\
\varepsilon_{i}=(0, \ldots, 0,1, \underbrace{0, \ldots, 0}_{i}) \in V_{n}, i \in\{0, \ldots, n-1\} .
\end{gathered}
$$

Каждый вектор $\alpha=\left(\alpha_{n-1}, \ldots, \alpha_{1}, \alpha_{0}\right) \in V_{n}$ будем отождествлять с числом $\widetilde{\alpha}=2^{n-1} \alpha_{n-1}+\ldots+2 \alpha_{1}+\alpha_{0}$. Таким образом, на $V_{n}$ можно определить операции сложения и умножения по модулю $2^{n}$. Эти операции обозначаются теми же символами, что и соответствующие операции над числами. Из контекста всегда будет ясно, о каких операциях идет речь.

Пусть $\alpha \in_{U} V_{n}$, а разностная характеристика $d$ преобразования $h_{1}, h_{2}$ определена входной и выходной разностями $\delta, \omega \in V_{n}$ соответственно. Тогда вероятность

$$
p=\mathbf{P}\left\{h_{1}(\alpha) \oplus h_{2}(\alpha \oplus \delta)=\omega\right\}
$$

будем называть вероятностью разностной характеристики $d$. Такую разностную характеристику $d$ будем обозначать следующим образом:

$$
\delta \stackrel{h_{1}, h_{2}, p}{\longrightarrow} \omega .
$$

Алгоритм шифрования ГОСТ 28147-89 основан на схеме Фейстеля, состоит из 32 раундов, размер блока текста равен 64 битам, длина ключа 256 бит.

Пусть $\alpha^{(0)}=\left(\alpha_{1}^{(0)}, \alpha_{0}^{(0)}\right)-$ открытый текст из $V_{32}^{2}, k=\left(\widehat{k}_{1}, \ldots, \widehat{k}_{8}\right)-$ ключ шифрования из $V_{32}^{8}$.

На $i$-м раунде раундовый ключ $\widehat{k}^{(i)} \in V_{32}$ задается равенствами

$$
\widehat{k}^{(i)}= \begin{cases}\widehat{k}_{i}, & \text { если } 1 \leq i \leq 8, \\ \widehat{k}_{i-8}, & \text { если } 9 \leq i \leq 16, \\ \widehat{k}_{i-16}, & \text { если } 16 \leq i \leq 24, \\ \widehat{k}_{33-i}, & \text { если } 25 \leq i \leq 32 .\end{cases}
$$
образом:

Пусть $\widehat{\beta} \in V_{32}$. Определим функцию $h_{\widehat{k}^{(i)}}: V_{32}^{2} \rightarrow V_{32}^{2}$ следующим

$$
h_{\widehat{k}^{(i)}}(\widehat{\beta})=\widehat{s}\left(\widehat{k}^{(i)}+2^{32} \widehat{\beta}\right) \lll 11 .
$$


Тогда действие раундовой функции $g_{\widehat{k}^{(i)}}: V_{32}^{2} \rightarrow V_{32}^{2}$ алгоритма шифрования ГОСТ 28147-89 представляется в виде

$$
\begin{gathered}
g_{\widehat{k}^{(i)}}\left(\alpha^{(i-1)}\right)=\alpha^{(i)}, \\
\alpha^{(i)}=\left(\widehat{\alpha}_{0}^{(i-1)}, \widehat{\alpha}_{1}^{(i-1)} \oplus h_{\widehat{k}^{(i)}}\left(\widehat{\alpha}_{0}^{(i-1)}\right)\right), \quad i \in\{1, \ldots, 32\} .
\end{gathered}
$$

Положим $k(j)=\left(\widehat{k}^{(j)}, \ldots, \widehat{k}^{(1)}\right), j=1, \ldots, 32$. Тогда функция зашифрования $f_{k(32)}: V_{32}^{2} \rightarrow V_{32}^{2}$ есть $f_{k(32)}=g_{\widehat{k}^{(32)}} \ldots g_{\widehat{k}^{(1)}}$.

Отметим, что финальная перестановка в описании алгоритма шифрования ГОСТ 28147-89 нами опущена, поскольку она не существенна для дальнейшего изложения.

В работе используются общепринятые терминология и подходы методов разностного, бумеранга и связанных ключей (см., например, [18-20]).

\section{3. Атака на алгоритм ГОСТ 28147-89 \\ с 12 связанными ключами}

В [15] приведена атака на алгоритм блочного шифрования ГОСТ 2814789 на основе метода бумеранга с 18 связанными ключами. При анализе данной работы были определены случаи, к которым приведенная в ней атака неприменима. Эта ситуация имеет место, если подстановка $\widetilde{s}_{8}$ обладает линейным транслятором $\widetilde{\varepsilon}_{3}$, т. е. выполнено соотношение

$$
\mathbf{P}\left\{\widetilde{s}_{8}(\widetilde{\alpha}) \oplus \widetilde{s}_{8}\left(\widetilde{\alpha} \oplus \widetilde{\varepsilon}_{3}\right)=\widetilde{\omega}\right\} \in\{0,1\}, \quad \widetilde{\alpha} \in_{U} V_{4}, \widetilde{\omega} \in V_{4}^{\times},
$$

в силу которого невозможно отличить истинные биты ключа от ложных с помощью представленного в [15] различителя. Атака не позволяет также найти раундовые ключи $25,26-г о$ раундов алгоритма блочного шифрования ГОСТ 28147-89.

В данном разделе приводится модификация атаки методом бумеранга со связанными ключами из [15], позволяющая восстанавливать все 256 бит ключа шифрования при произвольном $s$-боксе. В ряде случаев ключ шифрования можно найти на ЭВМ за приемлемое время. Кроме того, число связанных ключей уменьшается до 12. При наличии линейного транслятора у подстановки $\widetilde{S}_{8}$ для восстановления раундового ключа $i$-го раунда, $i \in\{27, \ldots, 32\}$, используется модификация различителя работы [15]. Для определения раундовых ключей 25 и 26-го раундов предложена новая разностная характеристика.

Рассматриваемый способ восстановления ключа шифрования состоит из двух основных этапов. На первом этапе определяются множества $K^{[32]}$, 
$K^{[31]}$ методом бумеранга со связанными ключами (используются четверки связанных ключей), на втором этапе находятся множества $K^{[30]}, \ldots, K^{[25]}$ разностным методом со связанными ключами (используются пары связанных ключей). Каждый из этапов предполагает наличие двух модификаций в зависимости от свойств подстановки $\widetilde{S}_{8}$.

\section{1. Нахождение множеств $K^{[32]}, K^{[31]}$}

В данном разделе рассматривается метод нахождения множеств $K^{[32]}, K^{[31]}$. Если подстановка $\widetilde{s}_{8}$ не обладает линейным транслятором, то множества $K^{[32]}, K^{[31]}$ определяются методом бумеранга со связанными ключами аналогично работе [15]. Если же подстановка $\widetilde{s}_{8}$ обладает линейным транслятором, то используется другой ключевой различитель, построенный с помощью следующего утверждения.

Утверждение 1. Если $\widetilde{s}_{8} \in S\left(V_{4}\right)$ обладает линейным транслятором $\widetilde{\varepsilon}_{3}$, то существует такая разность $\widetilde{\omega} \in V_{4}^{\times}$, что

$$
\mathbf{P}\left\{\widetilde{s}_{8}\left(\widetilde{\alpha}+{ }_{16} 1\right) \oplus \widetilde{s}_{8}(\widetilde{\alpha})=\widetilde{\omega}\right\}=2^{-3}, \widetilde{\alpha} \in_{U} V_{4} .
$$

Данное утверждение было доказано с помощью вычислительного эксперимента. При проведении эксперимента утверждение 1 было проверено для всех подстановок $\widetilde{s}_{8} \in S\left(V_{4}\right)$, обладающих фиксированным линейным транслятором $\widetilde{\varepsilon}_{3}$.

Далее приводятся утверждения 2 и 3, на которых основан алгоритм 3.1.1 нахождения множеств $K^{[r]}$ кандидатов в раундовые ключи $r$-го раунда при наличии множеств $K^{[32]}, \ldots, K^{[r+1]}$ в случаях, когда подстановка $\widetilde{s}_{8}$ обладает линейным транслятором $\widetilde{\varepsilon}_{3}, r \in\{27, \ldots, 32\}\left(K^{[33]}=\varnothing\right.$ при $r=32)$. На первых двух этапах алгоритма 3.1.1 производится восстановление старших трех бит раундового ключа с помощью утверждения 2, на третьем этапе - восстановление младших 27 бит на основе утверждения 3.

Утверждение 2. Пусть подстановка $\widetilde{s}_{8} \in S\left(V_{4}\right)$ обладает линейным транслятором $\widetilde{\varepsilon}_{3}, \widehat{k} \in V_{32}$ и вектор $\widehat{\gamma}_{0} \in V_{32}$ задан равенством

$$
\widehat{\gamma}_{0}=\left(\widehat{s}(\widehat{k}) \oplus \widehat{s}\left(\widehat{k} \oplus \widehat{\varepsilon}_{27}\right)\right) \lll 11 .
$$

Тогда существует такой вектор $\widetilde{\tau} \in V_{4}$, что при

$$
\begin{aligned}
& \widehat{\gamma}_{1}=\widehat{\gamma}_{0} \oplus\left(\widehat{s}\left(\left(\widetilde{\tau}, \widetilde{\varepsilon}_{3}, \widetilde{0}, \ldots, \widetilde{0}\right)+{ }_{2^{32}} \widehat{k}\right) \oplus\right. \\
&\left.\oplus \widehat{s}\left(\left(\widetilde{\tau}, \widetilde{\varepsilon}_{3}, \widetilde{0}, \ldots, \widetilde{0}\right)+{ }_{2^{32}}\left(\widehat{k} \oplus \widehat{\varepsilon}_{27}\right)\right)\right) \lll 11
\end{aligned}
$$


выполняются равенства

$$
\begin{gathered}
\widehat{\gamma}_{1}=(0, \ldots, 0, \widetilde{\delta}, \underbrace{0, \ldots, 0}_{7}) \in V_{32}, \quad \widetilde{\delta} \in V_{4}^{\times}, \\
\mathbf{P}\left\{\widetilde{s}_{8}\left(\widetilde{\tau}+_{16} \widetilde{\kappa}\right) \oplus \widetilde{s}_{8}\left(\widetilde{\tau}+{ }_{16} \widetilde{\kappa}+{ }_{16} 1\right)=\widetilde{\delta}\right\}=2^{-3}, \quad \widetilde{\kappa} \in V_{U} .
\end{gathered}
$$

Доказательство проводится непосредственной проверкой.

Утверждение 3. Пусть подстановка $\widetilde{s}_{8} \in S\left(V_{4}\right)$ обладает линейнымм транслятором $\widetilde{\varepsilon}_{3}, \widehat{k}=\left(k_{31}, \ldots, k_{0}\right) \in V_{32}, t \in\{0, \ldots, 26\}$, и вектор $\widehat{\kappa}=$ $=\left(\kappa_{31}, \ldots, \kappa_{0}\right) \in V_{32}$ определен равенством

$$
\kappa_{i}= \begin{cases}0, & \text { если } i>26 \text { или } i<t, \\ k_{i} \oplus 1, & \text { если } t<i \leq 26, \\ 1, & \text { если } i=t,\end{cases}
$$

авектор $\left(\widetilde{\gamma}_{1}, \widetilde{\gamma}_{0}\right) \in V_{8}-$ равенством

$$
\left(0, \ldots, 0, \widetilde{\gamma}_{1}, \widetilde{\gamma}_{0}, 0,0,0\right)=\left(\widehat{s}\left(\widehat{\kappa}+{ }_{2^{32}} \widehat{k}\right) \oplus \widehat{s}\left(\widehat{\kappa}+{ }_{2^{32}}\left(\widehat{k} \oplus \widehat{\varepsilon}_{27}\right)\right)\right) \lll 11
$$

Условие $\widetilde{\gamma}_{1}=\widetilde{0}$ выполняется тогда и только тогда, когда $k_{t}=0$.

Доказательство очевидно.

Для нахождения множества $K^{[r]}$ в алгоритме 3.1 .1 используется четверка связанных ключей $k, k^{\prime}, k^{\prime \prime}, k^{\prime \prime \prime} \in V_{256}$ вида

$$
\begin{aligned}
& k \oplus k^{\prime}=k^{\prime \prime} \oplus k^{\prime \prime \prime}=\left(\widehat{\varepsilon}_{31}, \widehat{0}, \widehat{\varepsilon}_{31}, \widehat{0}, \widehat{\varepsilon}_{31}, \widehat{0}, \widehat{\varepsilon}_{31}, \widehat{0}, \widehat{\varepsilon}_{31}, \widehat{0}\right), \\
& k \oplus k^{\prime \prime}=k^{\prime} \oplus k^{\prime \prime \prime}=(\underbrace{\widehat{0}, \ldots, \hat{0}}_{32-r}, \widehat{\varepsilon}_{27}, \underbrace{\widehat{0}_{1}, \ldots, \widehat{0}}_{r-25}) .
\end{aligned}
$$

На первом этапе алгоритма определяется значение $\widehat{\gamma}_{0}$, на втором находится множество $\Theta$ пар $(\widetilde{\tau}, \widetilde{\delta})$ в соответствии с утверждением 2 , после чего определяются три старших бита раундового ключа. Из утверждения 2 и того, что на первых двух этапах осуществляется перебор всех возможных значений $\widetilde{\gamma}, \widetilde{\tau}, \widetilde{\delta} \in V_{4}$, следует, что в результате выполнения этих этапов всегда найдутся вектор $\widehat{\gamma}_{0}$ и множество $\Theta \neq \emptyset$. Алгоритм 3.1.1 приведен в приложении А. Оценка трудоемкости алгоритма и необходимое число открытых текстов приведены в разделе 4.

2013. T. 4. № 2. C. 127-152 


\section{2. Нахождение множеств $K^{[30]}, \ldots, K^{[25]}$ кандидатов в раундо- вые ключи для раундов 30-25}

В данном разделе рассматриваются методы нахождения множества $K^{[r]}$ кандидатов в раундовые ключи $r$-го раунда при наличии множеств $K^{[32]}, \ldots, K^{[r+1]}, r \in\{25, \ldots, 30\}$, с помощью двух связанных ключей. Если подстановка $\widetilde{s}_{8}$ не обладает линейным транслятором, то для восстановления множеств $K^{[r]}, r \in\{25, \ldots, 30\}$, используется алгоритм 3.2.1. Он основан на ключевом различителе из работы [15] и разностной характеристике, приведенной в утверждении 4. В алгоритме 3.2.1 для нахождения множества $K^{[r]}$ используется пара таких связанных ключей $k, k^{\prime} \in V_{256}$, что

$$
k \oplus k^{\prime}=(\underbrace{\widehat{0}, \ldots, \widehat{0}}_{r-25}, \widehat{\varepsilon}_{31}, \widehat{\delta}, \widehat{\varepsilon}_{31}, \underbrace{\widehat{0}, \ldots, \widehat{0}}_{30-r}), \quad \widehat{\delta}=(0, \ldots, 0, \widetilde{\delta}, \underbrace{0, \ldots, 0}_{7}) .
$$

В следующем утверждении описана разностная характеристика, применяемая в алгоритме 3.2.1. Разность между связанными ключами задается таким образом, чтобы нулевая разность между соответствующими промежуточными текстами сохранялась после трех раундов шифрования с некоторой ненулевой вероятностью. Используя данное свойство, строится разностная характеристика для случаев $r \in\{25, \ldots, 30\}$.

Утверждение 4. Пусть $\alpha^{(0)}=\left(\widehat{\alpha}_{1}^{(0)}, \widehat{\alpha}_{0}^{(0)}\right) \in_{U} V_{32}^{2}, \widehat{k}^{(i)}, \widehat{k}^{\prime(i)} \in V_{32}$, $\widehat{\delta} \in V_{32}, i \in\{1,2,3\}$, и выполнены соотношения

$$
\widehat{k}^{(1)} \oplus \widehat{k}^{(1)}=\widehat{k}^{(3)} \oplus \widehat{k}^{(3)}=\widehat{\varepsilon}_{31}, \quad \widehat{k}^{(2)} \oplus \widehat{k}^{(2)}=\widehat{\delta} .
$$

Пусть также

$$
\alpha^{(3)}=g_{\widehat{k}^{(3)}} g_{\widehat{k}^{(2)}} g_{\widehat{k}^{(1)}}\left(\alpha^{(0)}\right), \quad \alpha^{\prime(3)}=g_{\widehat{k}^{\prime(3)}} g_{\widehat{k}^{\prime}(2)} g_{\widehat{k}^{\prime}(1)}\left(\alpha^{(0)}\right) .
$$

Тогда

$$
2^{-10} \leq \mathbf{P}\left\{\alpha^{(3)}=\alpha^{\prime(3)}\right\} \leq 2^{-1}
$$

Доказательство данного утверждения приводится в приложении Б.

Алгоритм 3.2.1 представлен в приложении В. Оценка трудоемкости алгоритма 3.2.1 приводится в разделе 4 . 
Если подстановка $\widetilde{s}_{8}$ обладает линейным транслятором, то для нахождения множеств $K^{[r]}, r \in\{25, \ldots, 30\}$, используется алгоритм 3.2 .2 с разностной характеристикой, аналогичной приведенной в утверждении 4. Его ключевой различитель основан на ключевом различителе алгоритма 3.1.1.

В алгоритме 3.2.2 для нахождения множества $K^{[r]}$ применяется такая пара связанных ключей $k, k^{\prime} \in V_{256}$, что

$$
k \oplus k^{\prime}=(\underbrace{\widehat{0}, \ldots, \widehat{0}}_{r-25}, \widehat{\varepsilon}_{27}, \widehat{\delta}^{\prime}, \widehat{\varepsilon}_{27}, \underbrace{\widehat{0}, \ldots, \widehat{0}}_{30-r}),
$$

где $\widehat{\delta}^{\prime}=\left(0, \ldots, 0, \widetilde{\delta}^{\prime}, 0,0,0\right), \widetilde{\delta}^{\prime} \in V_{4}^{\times}$.

Алгоритм 3.2.2 представлен в приложении Г, а его оценка трудоемкости приведена в разделе 4.

Таким образом, в зависимости от свойств подстановки $\widetilde{s}_{8}$ ключ шифрования находится с использованием 12 связанных ключей посредством алгоритмов І.1 и I.2.

Если $\widetilde{s}_{8}$ не обладает линейным транслятором $\widetilde{\varepsilon}_{3}$, то применяется алгоритм I.1, состоящий из следующих этапов:

1. Множества $K^{[32]}, K^{[31]}$ находятся с помощью алгоритма из [15].

2. Оставшиеся биты ключа шифрования восстанавливаются посредством алгоритма 3.2.1.

Если $\widetilde{s}_{8}$ обладает линейным транслятором $\widetilde{\varepsilon}_{3}$, то используется алгоритм I.2, состоящий из следующих этапов:

1. Для нахождения множеств $K^{[32]}, K^{[31]}$ применяется алгоритм 3.1.1.

2. Оставшиеся биты ключа шифрования находятся с помощью алгоритма 3.2.2.

\section{4. Оценки трудоемкости атак}

В данном разделе представлены оценки трудоемкости атак с использованием 12 связанных ключей.

2013. T. 4. № 2. C. 127-152 
Приведем сначала оценку трудоемкости алгоритма 3.1.1. В нем применены подходы, аналогичные атаке методом бумеранга со связанными ключами из [15], но выбран другой различитель ключа. Легко проверить, что алгоритм 3.1.1 использует

$$
\begin{gathered}
T_{32}^{(3.1 .1)}=2^{4} \cdot\left(4+\frac{1}{4}\right)+2^{8} \cdot\left(4+\frac{1}{4}\right)+27 \cdot 2^{8} \cdot\left(4+\frac{1}{4}\right) \approx 2^{14.97} \\
T_{31}^{(3.1 .1)}=2^{2} \cdot\left(2^{4} \cdot\left(4+\frac{1}{4}\right)+2^{8} \cdot\left(4+\frac{1}{4}\right)+27 \cdot 2^{8} \cdot\left(4+\frac{1}{4}\right)\right) \approx 2^{16.97}
\end{gathered}
$$

операций шифрования для 32 и 31 раундов соответственно, где $2^{4} \cdot 4$ - число шифрований на первом этапе, $2^{8} \cdot 4$ - на втором этапе, и $27 \cdot 2^{8} \cdot 4-$ на третьем этапе. Для успешного выполнения алгоритма 3.1.1 требуется

$$
\begin{gathered}
n_{0,32}^{(3.1 .1)}=\left(1+2^{4}\right)+\left(2^{4}+2^{8}\right)+\left(27+27 \cdot 2^{4}\right) \approx 2^{9.55}, \\
n_{0,31}^{(3.1 .1)}=2^{2} \cdot\left(\left(1+2^{4}\right)+\left(2^{4}+2^{8}\right)+\left(27+27 \cdot 2^{4}\right)\right) \approx 2^{11.55}
\end{gathered}
$$

выбранных шифртекстов для 32 и 31-го раундов соответственно, а вероятность успешного выполнения алгоритма равна единице.

Здесь и далее фраза «успешное выполнение алгоритма» означает, что алгоритм находит решение задачи. Соответственно, вероятность наступления данного события при случайном и равновероятном выборе входных параметров будем называть вероятностью успешного выполнения алгоритма.

Отметим, что

$$
\left|K^{[r]}\right|=2^{2} \cdot\left|K^{[r+1]}\right|, \quad r \in\{31,32\} \text {, и }\left|K^{[33]}\right|=1 .
$$

Рассмотрим теперь вероятностный алгоритм 3.2.1. В нем используются разностные соотношения из утверждения 4 и два связанных ключа. Пусть $p^{(3.2 .1)}-$ вероятность разностной характеристики, применяемой в алгоритме 3.2.1.

Поскольку разностная характеристика, заданная в утверждении 4, используется дважды, справедливо неравенство

$$
\left(2^{-10}\right)^{2} \cdot 2^{-1} \leq p^{(3.2 .1)} \leq\left(2^{-1}\right)^{2} \cdot 2^{-1} .
$$

Пусть $\delta^{(3.2 .1)}-$ вероятность успешного выполнения алгоритма 3.2.1, $\delta^{(3.2 .1)} \in(0,1)$. Равенство

$$
\delta^{(3.2 .1)}=\left(1-\left(1-p^{(3.2 .1)}\right)^{n_{0}^{\prime(3.2 .1)}}\right)^{\left(2^{4}+27\right)}
$$


определяет необходимое число $n_{0}^{\prime(3.2 .1)}$ различных пар опробуемых шифртекстов для получения пар, удовлетворяющих разностным характеристикам в соответствии с описанием алгоритма 3.2.1. Логарифмируя соотношение (1), получаем

$$
n_{0}^{\prime(3.2 .1)}\left(\delta^{(3.2 .1)}\right)=\log _{\left(1-p^{(3.2 .1)}\right)}\left(1-\sqrt[\left(2^{4}+27\right)]{\delta^{(3.2 .1)}}\right)
$$

Число $T^{(3.2 .1)}\left(\delta^{(3.2 .1)}\right)$ операций шифрования в алгоритме 3.2 .1 оценивается величиной $2^{17} n_{0}^{\prime(3.2 .1)}\left(\delta^{(3.2 .1)}\right)$, а число шифртекстов для успешного нахождения ключа - величиной $n_{0}^{(3.2 .1)}\left(\delta^{(3.2 .1)}\right)=2^{10} n_{0}^{\prime(3.2 .1)}\left(\delta^{(3.2 .1)}\right)$. Для оценки трудоемкости второго этапа алгоритма 3.2.1 используются оценки работы [15].

Аналогичным образом можно получить оценки для алгоритма 3.2.2:

$$
\begin{aligned}
& \left(2^{-10} \cdot 2^{-2}\right)^{2} \cdot 2^{-1} \leq p^{(3.2 .2)} \leq\left(2^{-1} \cdot 2^{-2}\right)^{2} \cdot 2^{-1}, \\
& n_{0}^{\prime(3.2 .2)}\left(\delta^{(3.2 .2)}\right)=\log _{\left(1-p^{(3.2 .2)}\right)}\left(1-\sqrt[\left(2^{4}+2^{8}+2^{8} \cdot 27\right)]{\delta^{(3.2 .2)}}\right), \\
& T^{(3.2 .2)}\left(\delta^{(3.2 .2)}\right)=2^{14.97} n_{0}^{\prime(3.2 .2)}\left(\delta^{(3.2 .2)}\right), \\
& n_{0}^{(3.2 .2)}\left(\delta^{(3.2 .2)}\right)=2^{9.55} n_{0}^{\prime(3.2 .2)}\left(\delta^{(3.2 .2)}\right), \\
& \left|K^{[r]}\right|=2^{2} \cdot\left|K^{[r+1]}\right|, r \in\{25, \ldots, 30\} .
\end{aligned}
$$

Пусть $\delta=1-10^{-3}-$ вероятность успеха обоих алгоритмов 3.2 .1 и 3.2.2. Тогда числа шифрований в алгоритмах I.1 и I.2 можно оценить величинами

$$
\begin{gathered}
T^{(I .1)}=\left(2^{17}+2 \cdot 2^{17}\right)+2 T^{(3.2 .1)} \cdot\left(1+2^{2}+2^{4}+2^{6}+2^{8}+2^{10}\right) \approx \\
\approx 3 \cdot 2^{17}+2^{11.41} T^{(3.2 .1)}, \\
T^{(I .2)}=\left(T_{32}^{(3.1 .1)}+T_{31}^{(3.1 .1)}\right)+2^{2} T^{(3.2 .2)} \cdot\left(1+2^{2}+2^{4}+2^{6}+2^{8}+2^{10}\right) \approx \\
\approx 5 \cdot 2^{14.97}+2^{12.41} T^{(3.2 .2)} .
\end{gathered}
$$

Следовательно,

$$
2^{34.73} \leq T^{(I .1)} \leq 2^{56.83}, \quad 2^{41.38} \leq T^{(I .2)} \leq 2^{63.39} .
$$

2013. T. 4. № 2. C. 127-152 
Приведем также оценки необходимого числа текстов, а также вероятности успеха для алгоритмов I.1 и I.2:

$$
\begin{gathered}
n^{(I .1)}=2^{10}+6 \cdot n^{(3.2 .1)}(\delta), n^{(I .2)}=n_{0,31}+n_{0,32}+6 \cdot n^{(3.2 .2)}(\delta) \\
2^{16.32} \leq n^{(I .1)} \leq 2^{38.42}, 2^{20.97} \leq n^{(I .2)} \leq 2^{42.98} \\
\delta^{(I .1)}=\delta^{6}=0.994, \delta^{(I .2)}=\delta^{8}=0.992 .
\end{gathered}
$$

\section{Список литературы}

1. Biham E., Dunkelman O., Keller N. Improved slide attacks // FSE-2007. Lect. Notes Comput. Sci. - 2007. - V. 459. - P. 153-166.

2. Biryukov A., Wagner D. Advanced slide attacks // Eurocrypt-2000.- Lect. Notes Comput. Sci. - 2000. - V. 1807. - P. 589-606.

3. Grossman E.K., Tuckerman B. Analysis of weakened Feistel like cipher // Proc. Int. Conf. on Communications. - Alger Press, 1978. - P. 46.3.1-46.3.5.

4. Kara O. Reflection cryptanalysis of some ciphers //INDOCRYPT-2008. Lect. Notes Comput. Sci. - 2008. - V. 5365. - P. 294-307.

5. Isobe T. A single-key attack on the full GOST block cipher // FSE-2011. Lect. Notes Comput. Sci., 2011. - V. 6733. - P. 290-305.

6. Courtois N. Security evaluation of GOST 28147-89 in view of international standardisation. - 2011. - http://eprint.iacr.org/2011/211.

7. Courtois N.T., Misztal M. Differential cryptanalysis of GOST. - 2011. http://eprint.iacr.org/2011/312.

8. Dinur I., Dunkelman O., Shamir A. Improved attacks on full GOST. - 2011. http://eprint.iacr.org/2011/558.

9. Courtois N. An improved differential attack on full GOST. - 2012. http://eprint.iacr.org/2012/138.

10. Zhu B., Gong G. Multidimensional meet-in-the-middle attack and its applications to GOST, KTANTAN and Hummingbird-2. - 2011. http://eprint.iacr.org/2011/619.

11. Kelsey J., Shneier B., Wagner D. Key-schedule cryptanalysis of IDEA, GDES, GOST, SAFER, and Triple-DES // CRYPTO'96. - Lect. Notes Comput. Sci. - 1996. - P. 237-251.

12. Seki H., Kaneko T. Differential cryptanalysis of reduced rounds of Gost // Selected areas in cryptography. - Lect. Notes Comput. Sci., Springer-Verlag. - 2000. - V. 2012. - P. 315-323. 
13. Ko Y., Hong S., Lee W., Lee S., Kang J.-S. Related key differential attacks on 27 rounds of Xtea and full-round Gost // FSE 2004. - Lect. Notes Comput. Sci. - 2004. - V. 3017. - P. 299-316.

14. Fleischmann E., Gorski M., Huhne J.-H., Lucks S. Key recovery attack on full GOST block cipher with zero time and memory // WEWoRC. - 2009.

15. Rudskoy $V$. On zero rractical significance of "key recovery attack on full GOST block cipher with zero time and memory". - 2010. http: //eprint.iacr.org/2010.

16. Pudovkina M., Khoruzhenko G. Related-key attacks on the full GOST block cipher with two or four related keys // BulCrypt-2012. - Sofia, 2012. P. 107-127.

17. Шнайер Б. Прикладная криптография. Протоколы, алгоритмы, исходные тексты на языке Си. - М.: Триумф, 2002.

18. Knudsen L.R. Cryptanalysis of LOKI91 // AUSCRYPT'92. - Lect. Notes Comput. Sci. - 1993. - V. 718. - P. 196-208.

19. Biham E., Shamir A. Differential cryptanalysis of the Data Encryption Standard. - Springer-Verlag, Berlin, 1993.

20. Wagner D. The boomerang attack // FSE'99. - Lect. Notes Comput. Sci. 1990. - V. 1636. - P. 156-170.

\section{5. Приложения}

\section{1. Приложение А. Алгоритм 3.1.1}

Здесь и далее под символом «:=» будем понимать оператор присваивания. В приведенном ниже алгоритме 3.1.1 для нахождения множества $K^{[r]}$ используется четверка связанных ключей $k, k^{\prime}, k^{\prime \prime}, k^{\prime \prime \prime} \in V_{256}$ вида

$$
\begin{aligned}
& k \oplus k^{\prime}=k^{\prime \prime} \oplus k^{\prime \prime \prime}=\left(\widehat{\varepsilon}_{31}, \widehat{0}, \widehat{\varepsilon}_{31}, \widehat{0}, \widehat{\varepsilon}_{31}, \widehat{0}, \widehat{\varepsilon}_{31}, \widehat{0}, \widehat{\varepsilon}_{31}, \widehat{0}\right), \\
& k \oplus k^{\prime \prime}=k^{\prime} \oplus k^{\prime \prime \prime}=(\underbrace{\widehat{0}, \ldots, \widehat{0}}_{32-r}, \widehat{\varepsilon}_{27}, \underbrace{\widehat{0}, \ldots, \widehat{0}}_{r-25}) .
\end{aligned}
$$

Алгоритм 3.1.1. Нахождение множества $K^{[r]}$ кандидатов в раундовые ключи r-го раунда методом бумеранга на связанных ключах.

Этап I. Нахождение разности $\widehat{\gamma}_{0} \in V_{32}^{\times}$.

Вход: $K^{[32]}, \ldots, K^{[r+1]}-$ множества кандидатов в раундовые ключи для раундов $32-(r+1)\left(K^{[33]}=\varnothing\right.$ при $\left.r=32\right)$.

Для всех $\widehat{k}^{(32)} \in K^{[32]}, \ldots, \widehat{k}^{(r+1)} \in K^{[r+1]}$ выполняем:

1. Полагаем $\widehat{\gamma}_{0}:=\widehat{0}$. 
2. Для всех $\widetilde{\gamma} \in V_{4}$ выполняем:

2.1. Полагаем

$$
\begin{gathered}
\alpha^{(32)}:=g_{\widehat{k}^{(32)}} \ldots g_{\widehat{k}^{(r+1)}}(\widehat{0}, \widehat{0}), \\
\alpha^{\prime(32)}:=g_{\widehat{k}^{\prime \prime(32)}} \ldots g_{\widehat{k}^{\prime \prime(r+1)}}(\widehat{0},(0, \ldots, 0, \widetilde{\gamma}, 0,0,0)) .
\end{gathered}
$$

2.2. Вычисляем

$$
\alpha^{(0)}:=f_{k}^{-1}\left(\alpha^{(32)}\right), \quad \alpha^{\prime(0)}:=f_{k^{\prime \prime}}^{-1}\left(\alpha^{\prime(32)}\right) .
$$

2.3. Полагаем

$$
\beta^{(0)}:=\alpha^{(0)} \oplus\left(\widehat{0}, \widehat{\varepsilon}_{31}\right), \quad \beta^{(0)}:=\alpha^{\prime(0)} \oplus\left(\widehat{0}, \widehat{\varepsilon}_{31}\right) .
$$

2.4. Вычисляем

$$
\beta^{(32)}:=f_{k^{\prime}}\left(\beta^{(0)}\right), \quad \beta^{\prime(32)}:=f_{k^{\prime \prime \prime}}\left(\beta^{\prime(0)}\right) .
$$

\section{5. Находим}

$$
\beta^{(r)}:=g_{\widehat{k}^{(r+1)}}^{-1}\left(\ldots g_{\widehat{k}^{(32)}}^{-1}\left(\beta^{(32)}\right)\right), \quad \beta^{\prime(r)}:=g_{\widehat{k}^{\prime}(r+1)}^{-1}\left(\ldots g_{\widehat{k}^{\prime}(32)}^{-1}\left(\beta^{\prime(32)}\right)\right) .
$$

2.6. Полагаем

$$
\widehat{\lambda}=\left(\widetilde{\lambda}_{7}, \ldots, \widetilde{\lambda}_{0}\right):=\left(\widehat{\beta}_{0}^{(r)} \oplus \widehat{\beta}_{0}^{(r)}\right) \ggg 3
$$

2.7. Если $\widehat{\beta}_{1}^{(r)} \oplus \widehat{\beta}_{1}^{(r)}=\widehat{0}$ и $\widetilde{\lambda}_{j}=\widetilde{0}, j=1, \ldots, 7$, то полагаем $\widehat{\gamma}_{0}:=$ $:=(0, \ldots, 0, \widetilde{\gamma}, 0,0,0)$ и завершаем работу алгоритма.

Выход: разность $\widehat{\gamma}_{0}$.

Этап II. Нахождение битов $\left(k_{31}^{(r)}, k_{30}^{(r)}, k_{29}^{(r)}, k_{28}^{(r)}\right)$ раундового ключа $\widehat{k}^{(r)}$. Вход: $K^{[32]}, \ldots, K^{[r+1]}-$ множества кандидатов в раундовые ключи для раундов $32-(r+1)$, разность $\widehat{\gamma}_{0} \in V_{32}$.

Для всех $\widehat{k}^{(32)} \in K^{[32]}, \ldots, \widehat{k}^{(r+1)} \in K^{[r+1]}$ выполняем: 
1. Полагаем $\widetilde{K}^{[r]}:=\varnothing, \Theta:=\varnothing$.

2. Для всех $\widetilde{\tau} \in V_{4}, \widetilde{\delta} \in V_{4}{ }^{\times}$выполняем:

2.1. Полагаем

$$
\alpha^{(32)}:=g_{\widehat{\vec{k}}^{(32)}}\left(\ldots g_{\widehat{\hat{k}}^{(r+1)}}\left((\widetilde{\tau}, \underbrace{\widetilde{0}, \ldots, \widetilde{0}}_{7}) \oplus \widehat{\varepsilon}_{27}, \widehat{0}\right) \ldots\right),
$$

$\alpha^{\prime(32)}:=g_{{\widehat{k^{\prime \prime}}}^{\prime \prime(32)}}\left(\ldots g_{\widehat{k}^{\prime \prime}(r+1)}\left((\widetilde{\tau}, \underbrace{\widetilde{0}, \ldots, \widetilde{0}}_{7}) \oplus \widehat{\varepsilon}_{27}, \widehat{\gamma}_{0} \oplus(0, \ldots, 0, \widetilde{\delta}, \underbrace{0, \ldots, 0}_{7})\right) \ldots\right)$.

\section{2. Вычисляем}

$$
\alpha^{(0)}:=f_{k}^{-1}\left(\alpha^{(32)}\right), \quad \alpha^{(0)}:=f_{k^{\prime \prime}}^{-1}\left(\alpha^{\prime(32)}\right) .
$$

2.3. Полагаем

$$
\beta^{(0)}:=\alpha^{(0)} \oplus\left(\widehat{0}, \widehat{\varepsilon}_{31}\right), \quad \beta^{(0)}:=\alpha^{\prime(0)} \oplus\left(\widehat{0}, \widehat{\varepsilon}_{31}\right) .
$$

\section{4. Вычисляем}

$$
\beta^{(32)}:=f_{k^{\prime}}\left(\beta^{(0)}\right), \quad \beta^{(32)}:=f_{k^{\prime \prime \prime}}\left(\beta^{\prime(0)}\right) .
$$

\section{5. Вычисляем}

$$
\beta^{(r)}:=g_{\widehat{k}^{(r+1)}}^{-1} \ldots g_{\widehat{k}^{(32)}}^{-1}\left(\beta^{(32)}\right), \quad \beta^{\prime(r)}:=g_{\widehat{k}^{\prime}(r+1)}^{-1} \ldots g_{\widehat{k}^{\prime}(32)}^{-1}\left(\beta^{\prime(32)}\right) .
$$

2.6. Находим

$$
\widehat{\lambda}=\left(\widetilde{\lambda}_{7}, \ldots, \widetilde{\lambda}_{0}\right):=\left(\widehat{\beta}_{0}^{(r)} \oplus \widehat{\beta}_{0}^{(\prime r)}\right) \ggg 3
$$

2.7. Если $\widehat{\beta}_{1}^{(r)} \oplus \widehat{\beta}_{1}^{(r)}=\widehat{0}$ и $\widetilde{\lambda}_{j}=\widetilde{0}, j=2, \ldots, 7$, то полагаем $\Theta:=$ $\Theta \cup\{(\widetilde{\tau}, \widetilde{\delta})\}$.

3. Для всех $\widetilde{\kappa}=\left(\widetilde{\kappa}_{3}, \widetilde{\kappa}_{2}, \widetilde{\kappa}_{1}, \widetilde{\kappa}_{0}\right) \in V_{4}$ выполняем:

3.1. Полагаем $v_{\widetilde{\kappa}}:=0$. 
3.2. Для всех $(\widetilde{\tau}, \widetilde{\delta}) \in \Theta$ выполняем: если

$$
\widetilde{s}_{8}\left(\widetilde{\tau}+{ }_{16} \widetilde{\kappa}\right) \oplus \widetilde{s}_{8}\left(\widetilde{\tau}+{ }_{16} \widetilde{\kappa}+{ }_{16} 1\right)=\widetilde{\delta},
$$

то $v_{\tilde{\kappa}}:=v_{\tilde{\kappa}}+1$.

3.3. Полагаем $\widetilde{K}^{[r]}:=\left\{\widetilde{\kappa} \in V_{4} \mid v_{\widetilde{\kappa}}=\max \left\{v_{\widetilde{\lambda}} \mid \widetilde{\lambda} \in V_{4}\right\}\right\}$.

Выход: множество $\widetilde{K}^{[r]}$.

Этап III. Нахождение битов $k_{0}^{(r)}, \ldots, k_{26}^{(r)}$ раундового ключа $\widehat{k}^{(r)}$.

Вход: $K^{[32]}, \ldots, K^{[r+1]}-$ множества кандидатов в раундовые ключи для раундов $32-(r+1)$.

1. Полагаем $\bar{k}:=\widehat{0}, \kappa:=\widehat{0}$.

2. Для всех $t \in\{0, \ldots, 26\}$ и для всех $\gamma_{3}=\left(\widetilde{\gamma}_{3,1}, \widetilde{\gamma}_{3,0}\right) \in V_{8}$ выполняем:

2.1. Полагаем

$$
\begin{gathered}
\alpha^{(32)}:=g_{\widehat{k}^{(32)}} \ldots g_{\widehat{k}^{(r+1)}}\left(\kappa \oplus \widehat{\varepsilon}_{26-t}, \widehat{0}\right), \\
\alpha^{\prime(32)}:=g_{\widehat{k}^{\prime \prime(32)}} \ldots g_{\widehat{k}^{\prime \prime(r+1)}}\left(\kappa \oplus \widehat{\varepsilon}_{26-t},(\underbrace{0, \ldots, 0}_{21}, \gamma_{3}, 0,0,0)\right) .
\end{gathered}
$$

2.2. Вычисляем

$$
\alpha^{(0)}:=f_{k}^{-1}\left(\alpha^{(32)}\right), \quad \alpha^{\prime(0)}:=f_{k^{\prime \prime}}^{-1}\left(\alpha^{\prime(32)}\right) .
$$

2.3. Полагаем

$$
\beta^{(0)}:=\alpha^{(0)} \oplus\left(\widehat{0}, \widehat{\varepsilon}_{31}\right), \quad \beta^{(0)}:=\alpha^{(0)} \oplus\left(\widehat{0}, \widehat{\varepsilon}_{31}\right) .
$$

2.4. Вычисляем

$$
\beta^{(32)}:=f_{k^{\prime}}\left(\beta^{(0)}\right), \quad \beta^{\prime(32)}:=f_{k^{\prime \prime \prime}}\left(\beta^{\prime(0)}\right) .
$$

2.5. Находим

$$
\beta^{(r)}:=g_{\widehat{k}^{(r+1)}}^{-1} \ldots g_{\widehat{k}^{(32)}}^{-1}\left(\beta^{(32)}\right), \quad \beta^{\prime(r)}:=g_{\widehat{k}^{\prime(r+1)}}^{-1} \ldots g_{\widehat{k}^{\prime}(32)}^{-1}\left(\beta^{\prime(32)}\right) .
$$


2.6. Полагаем

$$
\widehat{\lambda}=\left(\widetilde{\lambda}_{7}, \ldots, \widetilde{\lambda}_{0}\right):=\left(\widehat{\beta}_{0}^{(r)} \oplus \widehat{\beta}_{0}^{(r)}\right) \ggg 3 .
$$

2.7. Если $\widehat{\beta}_{1}^{(r)} \oplus \widehat{\beta}_{1}^{(r)}=\widehat{0}$ и $\widetilde{\lambda}_{j}=\widetilde{0}, j=2, \ldots, 7$, то

2.7.1. Если $\widetilde{\gamma}_{3,1}=0$, то $\widehat{\kappa}:=\widehat{\kappa} \oplus \widehat{\varepsilon}_{26-t}$, переходим на шаг III.2.1 с $t:=t+1$ и следующим значением $\gamma_{3}$.

2.7.2. Если $\widetilde{\gamma}_{3,1} \neq 0$, то $\bar{k}:=\bar{k} \oplus \widehat{\varepsilon}_{26-t}$, переходим на шаг III.2.1 с $t:=t+1$ и следующим значением $\gamma_{3}$.

3. Полагаем $K^{[r]}:=\left\{\left(\widetilde{\kappa}, 0, \bar{k}_{26}, \ldots, \bar{k}_{0}\right) \cup\left(\widetilde{\kappa}, 1, \bar{k}_{26}, \ldots, \bar{k}_{0}\right) \mid \widetilde{\kappa} \in \widetilde{K}^{[r]}\right\}$ и завершаем работу алгоритма.

Выход: множество $K^{[r]}$ кандидатов в раундовые ключи $r$-го раунда.

\section{2. Приложение Б. Доказательство утверждения 4.}

Пусть

$$
\begin{aligned}
& \alpha^{(1)}=\left(\widehat{\alpha}_{1}^{(1)}, \widehat{\alpha}_{0}^{(1)}\right)=g_{\widehat{k}^{(1)}}\left(\alpha^{(0)}\right), \alpha^{\prime(1)}=\left(\widehat{\alpha}_{1}^{(1)}, \widehat{\alpha}_{0}^{\prime(1)}\right)=g_{\widehat{k}^{(1)} \oplus \widehat{\varepsilon}_{31}}\left(\alpha^{(0)}\right), \\
& \alpha^{(2)}=\left(\widehat{\alpha}_{1}^{(2)}, \widehat{\alpha}_{0}^{(2)}\right)=g_{\widehat{k}^{(2)}}\left(\alpha^{(1)}\right), \alpha^{\prime(2)}=\left(\widehat{\alpha}_{1}^{(2)}, \widehat{\alpha}_{0}^{\prime(2)}\right)=g_{\widehat{k}^{(2)} \oplus \widehat{\delta}}\left(\alpha^{\prime(1)}\right), \\
& \alpha^{(3)}=\left(\widehat{\alpha}_{1}^{(3)}, \widehat{\alpha}_{0}^{(3)}\right)=g_{\widehat{k}^{(3)}}\left(\alpha^{(2)}\right), \alpha^{\prime(3)}=\left(\widehat{\alpha}_{1}^{(3)}, \widehat{\alpha}_{0}^{\prime(3)}\right)=g_{\widehat{k}^{(3)} \oplus \widehat{\varepsilon}_{31}}\left(\alpha^{\prime(2)}\right) .
\end{aligned}
$$

Очевидно, что

$$
\begin{array}{ll}
\widehat{\alpha}_{1}^{(1)}=\widehat{\alpha}_{0}^{(0)}, & \widehat{\alpha}_{1}^{\prime(1)}=\widehat{\alpha}_{0}^{(0)}, \\
\widehat{\alpha}_{1}^{(2)}=\widehat{\alpha}_{0}^{(1)}, & \widehat{\alpha}_{1}^{(2)}=\widehat{\alpha}_{0}^{(1)}, \\
\widehat{\alpha}_{1}^{(3)}=\widehat{\alpha}_{0}^{(2)}, & \widehat{\alpha}_{1}^{(3)}=\widehat{\alpha}_{0}^{(2)} .
\end{array}
$$

Рассмотрим разностную характеристику $d$ вида

$$
(\widehat{0}, \widehat{0}) \stackrel{g_{\hat{k}^{\prime}(1)}, g_{\hat{k}^{\prime}(1)}, p_{1}}{\longrightarrow}(\widehat{0}, \widehat{\delta}) \stackrel{g_{\hat{k}^{\prime}(2)}, g_{\hat{k}^{\prime}(2)}, p_{2}}{\longrightarrow}(\widehat{\delta}, \widehat{0}) \stackrel{g_{\widehat{k}}(3), g_{\hat{k}^{\prime}(3)}, p_{3}}{\longrightarrow}(\widehat{0}, \widehat{0})
$$

Имеют место следующие соотношения:

$$
\begin{aligned}
p_{1}= & \mathbf{P}\left\{\widehat{s}\left(\widehat{\alpha}_{0}^{(0)}+{ }_{2^{32}} \widehat{k}^{(1)}\right) \oplus \widehat{s}\left(\widehat{\alpha}_{0}^{(0)}+{ }_{2^{32}}\left(\widehat{k}^{(1)} \oplus \widehat{\varepsilon}_{31}\right)\right)=\widehat{\delta} \ggg 11\right\}= \\
& =\mathbf{P}\left\{\tilde{s}_{8}\left(\tilde{\alpha}_{7}^{(0)}+{ }_{16} \tilde{k}_{7}^{(1)}\right) \oplus \tilde{s}_{8}\left(\tilde{\alpha}_{7}^{(0)}+{ }_{16}\left(\tilde{k}_{7}^{(1)} \oplus \tilde{\varepsilon}_{3}\right)\right)=\tilde{\delta}\right\}, \\
p_{3}= & \mathbf{P}\left\{\widehat{s}\left(\widehat{\alpha}_{0}^{(2)}+{ }_{2^{32}} \widehat{k}^{(3)}\right) \oplus \widehat{s}\left(\widehat{\alpha}_{0}^{(2)}+{ }_{2^{32}}\left(\widehat{k}^{(3)} \oplus \widehat{\varepsilon}_{31}\right)\right)=\widehat{\delta} \ggg 11\right\}= \\
& =\mathbf{P}\left\{\tilde{s}_{8}\left(\tilde{\alpha}_{7}^{(2)}+{ }_{16} \tilde{k}_{7}^{(3)}\right) \oplus \tilde{s}_{8}\left(\tilde{\alpha}_{7}^{(2)}+{ }_{16}\left(\tilde{k}_{7}^{(3)} \oplus \tilde{\varepsilon}_{3}\right)\right)=\tilde{\delta}\right\} .
\end{aligned}
$$

2013. T. 4. № 2. C. 127-152 


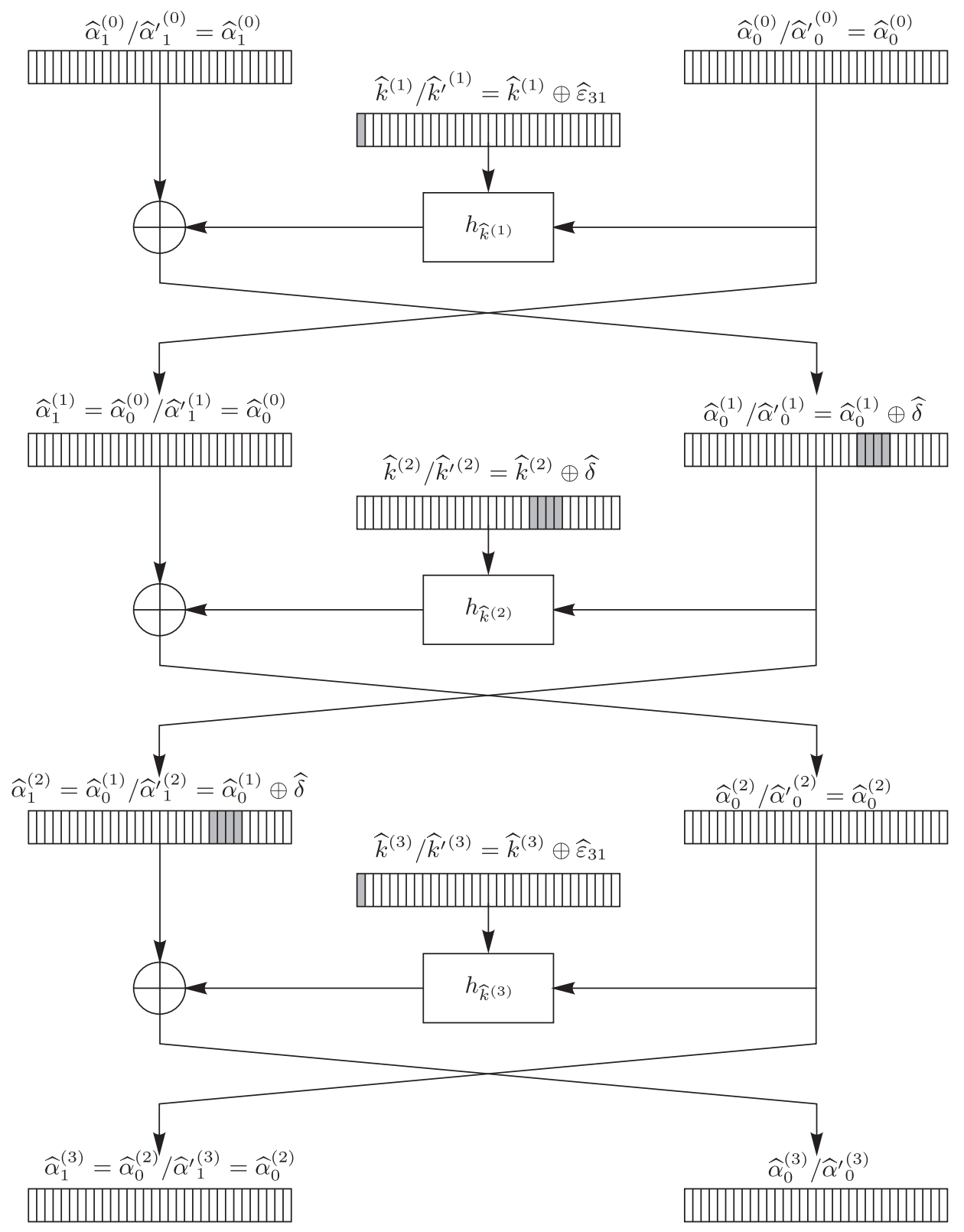

Рис. 1. Разностная характеристика трехраундового алгоритма шифрования ГОСТ 28147-89 с двумя связанными ключами 
Поскольку рассматриваемая разностная характеристика предполагает наличие только одной активной подстановки $s$-бокса $\tilde{s}_{8}$, то для вероятностей $p_{1}, p_{3}$ справедливы следующие оценки:

$$
2^{-3} \leq p_{1} \leq 1, \quad 2^{-3} \leq p_{3} \leq 1 .
$$

Так как вероятность $p_{2}$ есть

$$
\begin{aligned}
p_{2}= & \mathbf{P}\left\{\widehat{s}\left(\widehat{\alpha}_{0}^{(1)}+{ }_{2^{32}} \widehat{k}^{(2)}\right) \lll 11=\widehat{s}\left(\left(\widehat{\alpha}_{0}^{(1)} \oplus \widehat{\delta}\right)+_{2^{32}}\left(\widehat{k}^{(2)} \oplus \widehat{\delta}\right)\right) \lll 11\right\}= \\
& =\mathbf{P}\left\{\widehat{s}\left(\widehat{\alpha}_{0}^{(1)}+{ }_{2^{32}} \widehat{k}^{(2)}\right)=\widehat{s}\left(\left(\widehat{\alpha}_{0}^{(1)} \oplus \widehat{\delta}\right)+{ }_{2^{32}}\left(\widehat{k}^{(2)} \oplus \widehat{\delta}\right)\right)\right\}=2^{-\|\widehat{\delta}\|},
\end{aligned}
$$

Tо

$$
2^{-4} \leq p_{2} \leq 2^{-1}
$$

Справедливо равенство

$$
\mathbf{P}\left\{\alpha^{(3)}=\alpha^{\prime(3)}\right\}=p_{1} \cdot p_{2} \cdot p_{3} .
$$

Таким образом, из неравенств (2), (3) и соотношения (4) получаем

$$
2^{-10} \leq \mathbf{P}\left\{\alpha^{(3)}=\alpha^{(3)}\right\} \leq 2^{-1} .
$$

Рисунок 1 иллюстрирует рассматриваемую разностную характеристику.

\section{3. Приложение В. Алгоритм 3.2.1}

Будем использовать следующие обозначения. Для $i \in\{0, \ldots, 27\}$ положим

$$
\Omega\left(i, \widehat{k}^{(r)}\right)=V_{4} \times\left\{\left(\lambda_{27}, \ldots, \lambda_{0}\right)\right\},
$$

где

$$
\lambda_{t}= \begin{cases}0, & \text { если } t \in\{0, \ldots, i-1\} \\ 1, & \text { если } t=i, \\ k_{t}^{(r)} \oplus 1, & \text { если } t \in\{i+1, \ldots, 27\} .\end{cases}
$$

В данном разделе для нахождения множества $K^{[r]}$ алгоритмом 3.2 .1 используется пара таких связанных ключей $k, k^{\prime} \in V_{256}$, что $k \oplus k^{\prime}=(\underbrace{\widehat{0}, \ldots, \hat{0}}_{r-25}, \widehat{\varepsilon}_{31}, \widehat{\delta}, \widehat{\varepsilon}_{31}, \underbrace{\widehat{0}, \ldots, \hat{0}}_{30-r}), \widehat{\delta}=(0, \ldots, 0, \widetilde{\delta}, \underbrace{0, \ldots, 0}_{7}), \tilde{\delta} \in V_{4}^{\times}$. 
Алгоритм 3.2.1. Нахождение множества $K^{[r]}$ кандидатов в раундовые ключи для r-го раунда методом бумеранга на связанных ключах.

Этап I. Нахождение бит $\left(k_{30}^{(r)}, k_{29}^{(r)}, k_{28}^{(r)}\right)$ раундового ключа $\widehat{k}^{(r)}$.

Вход: $n_{0}^{(3.2 .1)}$ открытых текстов $\alpha^{(30-r, 1)}, \ldots, \alpha^{\left(30-r, n_{0}^{(3.2 .1)}\right)}$, $K^{[32]}, \ldots, K^{[r+1]}-$ множества кандидатов в раундовые ключи для раундов $32-(r+1)\left(K^{[33]}=\varnothing\right.$ при $\left.r=32\right)$.

1. Полагаем $\Theta:=\varnothing$.

2. Полагаем

$$
\begin{gathered}
\alpha^{(0, i)}:=g_{\widehat{k}^{(1)}}^{-1} \ldots g_{\widehat{k}^{(29-r)}}^{-1}\left(\alpha^{(30-r, i)}\right), \\
\alpha^{(0, i)}:=g_{\widehat{k}^{(1)}}^{-1} \ldots g_{\widehat{k}^{(29-r)}}^{-1}\left(\alpha^{(30-r, i)} \oplus(\widehat{\delta}, \widehat{0})\right), \quad i \in\left\{1, \ldots, n_{0}^{(3.2 .1)}\right\} .
\end{gathered}
$$

3. Вычисляем

$$
\alpha^{(32, i)}:=f_{k}\left(\alpha^{(0, i)}\right), \quad \alpha^{(32, i)}:=f_{k^{\prime}}\left(\alpha^{(0, i)}\right), \quad i \in\left\{1, \ldots, n_{0}^{(3.2 .1)}\right\} .
$$

4. Полагаем

$$
\begin{gathered}
\alpha^{(r, i)}:=g_{\widehat{k}^{(r+1)}}^{-1} \ldots g_{\widehat{k}^{(32)}}^{-1}\left(\alpha^{(32, i)}\right), \\
\alpha^{\prime(r, i)}:=g_{\widehat{k}^{(r+1)}}^{-1} \ldots g_{\widehat{k}^{(32)}}^{-1}\left(\alpha^{\prime(32, i)}\right), \quad i \in\left\{1, \ldots, n_{0}^{(3.2 .1)}\right\} .
\end{gathered}
$$

5. Если

$$
\alpha^{(r, i)} \oplus \alpha^{\prime(r, i)} \in\left\{(\widehat{0},(0, \ldots, 0, \widetilde{\lambda})) \in V_{32}^{2} \mid \widetilde{\lambda} \in V_{4}\right\},
$$

то полагаем $\Theta:=\Theta \cup\left(\alpha^{(30-r, i)}, \alpha^{(30-r, i)}\right), i \in\left\{1, \ldots, n_{0}^{(3.2 .1)}\right\}$.

6. Опробование бит $\left(k_{31}^{(r)}, k_{30}^{(r)}, k_{29}^{(r)}, k_{28}^{(r)}\right)$.

Для каждого $\widetilde{\kappa}=\left(\kappa_{3}, \kappa_{2}, \kappa_{1}, \kappa_{0}\right) \in V_{4}$ выполняем:

6.1. Полагаем $v_{\widetilde{\kappa}}:=0, \bar{k}:=\kappa_{3} \widehat{\varepsilon}_{31} \oplus \kappa_{2} \widehat{\varepsilon}_{30} \oplus \kappa_{1} \widehat{\varepsilon}_{29} \oplus \kappa_{0} \widehat{\varepsilon}_{28}, \bar{k}^{\prime}:=\bar{k} \oplus \widehat{\varepsilon}_{31}$.

6.2. Для каждого $\left(\alpha^{(30-r)}, \alpha^{\prime(30-r)}\right) \in \Theta$ выполняем:

6.2.1. Полагаем $\alpha^{(31-r)}:=g_{\bar{k}}\left(\alpha^{(30-r)}\right), \alpha^{\prime(31-r)}:=g_{\bar{k}^{\prime}}\left(\alpha^{\prime(30-r)}\right)$.

6.2.2. Если $\alpha^{(31-r)} \oplus \alpha^{\prime(31-r)}=(\widehat{0}, \widehat{0})$, то $v_{\widetilde{\kappa}}:=v_{\widetilde{\kappa}}+1$. 
7. Полагаем $\left(k_{30}^{(r)}, k_{29}^{(r)}, k_{28}^{(r)}\right):=\left(\kappa_{2}, \kappa_{1}, \kappa_{0}\right)$, где

$$
\max \left\{v_{\left(0, \kappa_{2}, \kappa_{1}, \kappa_{0}\right)}, v_{\left(1, \kappa_{2}, \kappa_{1}, \kappa_{0}\right)}\right\}=\max \left\{v_{\widetilde{\kappa}^{\prime}} \mid \widetilde{\kappa}^{\prime} \in V_{4}\right\} .
$$

Этап II. Нахождение битов $\left(k_{27}^{(r)}, \ldots, k_{0}^{(r)}\right)$ раундового ключа $\widehat{k}^{(r)}$.

Вход: $\left(k_{30}^{(r)}, k_{29}^{(r)}, k_{28}^{(r)}\right)-$ биты раундового ключа $\widehat{k}^{(r)}$.

1. Полагаем $t:=0$.

2. Полагаем $\left(k_{31}^{(r, t)}, k_{30}^{(r, t)}, k_{29}^{(r, t)}, k_{28}^{(r, t)}\right):=\left(t, k_{30}^{(r)}, k_{29}^{(r)}, k_{28}^{(r)}\right)$ и $v_{t}:=27$.

3. Рассматриваем $n_{0}^{(3.2 .1)}$ таких различных открытых текстов $\alpha^{(30-r, 1)}, \ldots, \alpha^{\left(30-r, n_{0}^{(3.2 .1)}\right)} \in V_{32}^{2}$, что

$$
\begin{gathered}
\left\{\widehat{\alpha}_{0}^{(30-r, j)} \mid j \in\left\{1, \ldots, n_{0}^{(3.2 .1)}\right\}\right\}=\Omega\left(v_{t}, \widehat{k}^{(30-r, t)}\right), \\
\left|\left\{\widehat{\alpha}_{1}^{(32, j)} \mid j \in\left\{1, \ldots, n_{0}^{(3.2 .1)}\right\}\right\}\right|=2^{6}
\end{gathered}
$$

где

$$
\alpha^{(30-r, j)}=\left(\widehat{\alpha}_{1}^{(30-r, j)}, \widehat{\alpha}_{0}^{(30-r, j)}\right), \quad j \in\left\{1, \ldots, n_{0}^{(3.2 .1)}\right\},
$$

т. е. $\widehat{\alpha}_{0}^{(30-r, j)} \in \Omega\left(v_{t}, \widehat{k}^{(30-r, t)}\right), \widehat{\alpha}_{1}^{(30-r, j)} \in_{U} V_{32}$

и $\left|\left\{\widehat{\alpha}_{1}^{(30-r, 1)}, \ldots, \widehat{\alpha}_{1}^{\left(30-r, n_{0}^{(3.2 .1)}\right)}\right\}\right|=2^{6}$.

4. Полагаем $\Theta_{v_{t}}:=\varnothing$.

5. Полагаем

$$
\begin{gathered}
\alpha^{(0, i)}:=g_{\widehat{k}^{(1)}}^{-1} \ldots g_{\widehat{k}^{(29-r)}}^{-1}\left(\alpha^{(30-r, i)}\right), \\
\alpha^{\prime(0, i)}:=g_{\widehat{k}^{(1)}}^{-1} \ldots g_{\widehat{k}^{(29-r)}}^{-1}\left(\alpha^{(30-r, i)} \oplus(\widehat{\delta}, \widehat{0})\right), \quad i \in\left\{1, \ldots, n_{0}^{(3.2 .1)}\right\} .
\end{gathered}
$$

6. Вычисляем

$$
\alpha^{(32, i)}:=f_{k}\left(\alpha^{(0, i)}\right), \quad \alpha^{\prime(32, i)}:=f_{k^{\prime}}\left(\alpha^{(0, i)}\right), \quad i \in\left\{1, \ldots, n_{0}^{(3.2 .1)}\right\} .
$$

7. Полагаем

$$
\begin{gathered}
\alpha^{(r, i)}:=g_{\widehat{k}^{(r+1)}}^{-1} \ldots g_{\widehat{k}^{(32)}}^{-1}\left(\alpha^{(32, i)}\right), \\
\alpha^{\prime(r, i)}:=g_{\widehat{k}^{(r+1)}}^{-1} \ldots g_{\widehat{k}^{(32)}}^{-1}\left(\alpha^{\prime(32, i)}\right), \quad i \in\left\{1, \ldots, n_{0}^{(3.2 .1)}\right\} .
\end{gathered}
$$

2013. T. 4. № 2. C. 127-152 
8. Если $\alpha^{(r, i)} \oplus \alpha^{\prime(r, i)} \in\left\{(\widehat{0},(0, \ldots, 0, \tilde{\lambda})) \in V_{32}^{2} \mid \widetilde{\lambda} \in V_{4}\right\}$, то

$$
\Theta_{v_{t}}:=\Theta_{v_{t}} \cup\left\{\left(\alpha^{(30-r, i)}, \alpha^{(30-r, i)}\right)\right\}, \quad i \in\left\{1, \ldots, n_{0}^{(3.2 .1)}\right\} .
$$

9. Для всех $i \in\{0,1\}$ находим $\widehat{u}^{(i)}=\left(u_{31}^{(i)}, \ldots, u_{0}^{(i)}\right) \in V_{32}$, где

$$
u_{j}^{(i)}= \begin{cases}0, & \text { если } j \in\left\{0, \ldots, v_{t}-1\right\} \\ i, & \text { если } j=v_{t} \\ k_{j}^{(r, t)}, & \text { если } j \in\left\{v_{t}+1, \ldots, 31\right\} .\end{cases}
$$

10. Полагаем $\widehat{u}^{(i)}:=\widehat{u}^{(i)} \oplus \widehat{\varepsilon}_{31}$ для $i=0,1$.

11. Для всех $i \in\{0,1\}$ выполняем:

11.1. Полагаем $b_{i}:=0$.

11.2. Для всех $\left(\alpha^{(30-r)}, \alpha^{\prime(30-r)}\right) \in \Theta_{v_{t}}$ выполняем:

11.2.1. Полагаем $\alpha^{(31-r)}:=g_{\widehat{u}^{(i)}}\left(\alpha^{(30-r)}\right), \alpha^{\prime(31-r)}:=g_{\widehat{u}^{\prime(i)}}\left(\alpha^{\prime(30-r)}\right)$.

11.2.2. Если $\alpha_{31}^{(31)} \oplus \beta_{31}^{(31)}=(\widehat{0}, \widehat{0})$, то $b_{i}:=b_{i}+1$.

12. Полагаем $k_{v_{t}}^{(r, t)}:=j$, где $b_{j}=\max \left\{b_{0}, b_{1}\right\}, j=0,1$.

13. Если $v_{t}>0$, то $v_{t}:=v_{t}-1$ и переходим к шагу II.3. Если $t=0, v_{t}=0$, то $t:=1$, и переходим к шагу II.2. Если $t=1, v_{t}=0$, то завершаем работу алгоритма.

Выход: $K^{[r]}=\left\{\widehat{k}^{(r, 0)}, \widehat{k}^{(r, 1)}\right\}$.

\section{4. Приложение Г. Алгоритм 3.2.2}

Приведенный ниже алгоритм является вероятностым. Величина $c \in\{0,1\}$ определяет успех его выполнения.

В данном разделе для нахождения множества $K^{[r]}$ используется пара таких связанных ключей $k, k^{\prime} \in V_{256}$, что

$k \oplus k^{\prime}=(\underbrace{\widehat{0}, \ldots, \widehat{0}}_{r-25}, \widehat{\varepsilon}_{27}, \widehat{\delta}^{\prime}, \widehat{\varepsilon}_{27}, \underbrace{\widehat{0}, \ldots, \widehat{0}}_{30-r}), \quad \widehat{\delta}^{\prime}=\left(0, \ldots, 0, \widetilde{\delta}^{\prime}, 0,0,0\right), \quad \widetilde{\delta}^{\prime} \in V_{4}^{\times}$. 
Алгоритм 3.2.2. Нахождение множества $K^{[r]}$ кандидатов в раундовые ключи для r-го раунда методом бумеранга на связанных ключах.

Этап I. Нахождение $\widehat{\gamma}_{0} \in V_{32}$.

Вход: $K^{[32]}, \ldots, K^{[r+1]}-$ множества кандидатов в раундовые ключи для раундов $32-(r+1)\left(K^{[33]}=\varnothing\right.$ при $\left.r=32\right)$.

Для всех $\widehat{k}^{(32)} \in K^{[32]}, \ldots, \widehat{k}^{(r+1)} \in K^{[r+1]}$ выполняем:

1. Полагаем $\widehat{\gamma}_{0}:=\widehat{0}, c:=0$.

2. Для всех $\widetilde{\gamma} \in V_{4}, i \in\left\{1, \ldots, n_{0}^{(3.2 .2)}\right\}$ выполняем:

2.1. Полагаем

$$
\begin{gathered}
\alpha^{(0, i)}:=g_{\widehat{k}^{(1)}} \ldots g_{\widehat{k}^{(32-r)}}\left(\widehat{0}, \alpha_{0}^{(32-r, i)}\right) \\
\alpha^{\prime(0, i)}:=g_{\widehat{k}^{\prime(1)}} \ldots g_{\widehat{k}^{\prime}(32-r)}\left(\widehat{0}, \alpha_{0}^{(32-r, i)} \oplus(0, \ldots, 0, \widetilde{\gamma}, 0,0,0)\right) .
\end{gathered}
$$

2.2. Вычисляем

$$
\alpha^{(32, i)}:=f_{k}\left(\alpha^{(0, i)}\right), \alpha^{\prime(32, i)}:=f_{k^{\prime}}\left(\alpha^{\prime(0, i)}\right) .
$$

2.3. Полагаем

$$
\begin{aligned}
\alpha^{(r, i)} & :=g_{\widehat{k}^{(r+1)}}^{-1}\left(\ldots g_{\widehat{k}^{(32)}}^{-1}\left(\alpha^{(32, i)}\right)\right), \\
\alpha^{\prime(r, i)} & :=g_{\widehat{k}^{\prime(r+1)}}^{-1}\left(\ldots g_{\widehat{k}^{\prime(32)}}^{-1}\left(\alpha^{\prime(32, i)}\right)\right) .
\end{aligned}
$$

2.4. Вычисляем

$$
\widehat{\lambda}=\left(\widetilde{\lambda}_{7}, \ldots, \widetilde{\lambda}_{0}\right):=\left(\widehat{\alpha}_{0}^{(r, i)} \oplus \widehat{\alpha}_{0}^{\prime(r, i)}\right) \ggg 3 .
$$

2.5. Если $\widehat{\alpha}_{1}^{(r, i)} \oplus \widehat{\alpha}_{1}^{\prime(r, i)}=\widehat{0}$ и $\widetilde{\lambda}_{j}=\widetilde{0}, j=1, \ldots, 7$, то полагаем

$$
\widehat{\gamma}_{0}:=(0, \ldots, 0, \widetilde{\gamma}, 0,0,0), \quad c:=1
$$

и завершаем работу алгоритма.

Выход: разность $\widehat{\gamma}_{0} \in V_{32}$, величина $c$.

Если $c=1$, то переходим к этапу II.

Этап II. Нахождение битов $\left(k_{31}^{(r)}, k_{30}^{(r)}, k_{29}^{(r)}, k_{28}^{(r)}\right)$ раундового ключа $\widehat{k}^{(r)}$. Вход: $K^{[32]}, \ldots, K^{[r+1]}-$ множества кандидатов в раундовые ключи для раундов 32- $(r+1)$, разность $\widehat{\gamma}_{0} \in V_{32}$.

Для всех $\widehat{k}^{(32)} \in K^{[32]}, \ldots, \widehat{k}^{(r+1)} \in K^{[r+1]}$ выполняем: 
1. Полагаем $\widetilde{K}^{[r]}:=\varnothing, \Theta:=\varnothing, c_{0}:=0$.

2. Для всех $\widetilde{\tau} \in V_{4}$ выполняем:

2.1. Полагаем $c:=0$.

2.2. Для всех $i \in\left\{1, \ldots, n_{0}{ }^{(3.2 .2)}\right\}$ выполняем:

2.2.1. Для всех $\widetilde{\delta} \in V_{4}$ выполняем:

2.2.1.1. Полагаем

$$
\begin{gathered}
\left.\alpha^{(0, i):=g_{\widehat{k}^{(1)}}\left(\ldots g_{\widehat{k}^{(32-r)}}\right.}\left(\left((\widetilde{\tau}, \underbrace{\widetilde{0}, \ldots, \widetilde{0}}_{7}) \oplus \widehat{\varepsilon}_{27}, \alpha_{0}^{(32-r, i)}\right)\right) \ldots\right), \\
\alpha^{\prime(0, i)}:=g_{\widehat{k}^{\prime(1)}}\left(\ldots \left(g _ { \widehat { k } ^ { \prime } ( 3 2 - r ) } \left(\left((\widetilde{\tau}, \underbrace{\widetilde{0}, \ldots, \widetilde{0}}_{7}) \oplus \widehat{\varepsilon}_{27}, \alpha_{0}^{(32-r, i)} \oplus\right.\right.\right.\right. \\
\left.\left.\left.\oplus \widehat{\gamma}_{0} \oplus(0, \ldots, 0, \widetilde{\delta}, \underbrace{0, \ldots, 0}_{7})\right) \ldots\right)\right) .
\end{gathered}
$$

2.2.1.2. Вычисляем

$$
\alpha^{(32, i)}:=f_{k}\left(\alpha^{(0, i)}\right), \alpha^{\prime(32, i)}:=f_{k^{\prime}}\left(\alpha^{(0, i)}\right) .
$$

2.2.1.3. Полагаем

$$
\begin{aligned}
\alpha^{(r, i)} & :=g_{\widehat{k}^{(r+1)}}^{-1}\left(\ldots\left(g_{\widehat{k}^{(32)}}^{-1}\left(\alpha^{(32, i)}\right)\right) \ldots\right), \\
\alpha^{\prime(r, i)} & :=g_{\widehat{k}^{\prime(r+1)}}^{-1}\left(\ldots\left(g_{\widehat{k}^{\prime(32)}}^{-1}\left(\alpha^{\prime(32, i)}\right)\right) \ldots\right) .
\end{aligned}
$$

2.2.1.4. Вычисляем

$$
\widehat{\lambda}=\left(\widetilde{\lambda}_{7}, \ldots, \widetilde{\lambda}_{0}\right):=\left(\widehat{\alpha}_{0}^{(r, i)} \oplus \widehat{\alpha}_{0}^{\prime(r, i)}\right) \ggg 3 .
$$

2.2.1.5. Если $\widehat{\alpha}_{1}^{(r, i)} \oplus \widehat{\alpha}_{1}^{\prime(r, i)}=\widehat{0}$ и $\widetilde{\lambda}_{j}=0, j=2, \ldots, 7$, то полагаем

$$
\Theta:=\Theta \cup\{(\tilde{\tau}, \tilde{\delta})\}, \quad c:=1 .
$$

2.2.2. Если $c=0$, то полагаем $\left(\widetilde{K}^{[r]}, c_{0}\right)=(\varnothing, 0)$ и завершаем работу алгоритма.

2.3. Для всех $\widetilde{\kappa}=\left(\widetilde{\kappa}_{3}, \widetilde{\kappa}_{2}, \widetilde{\kappa}_{1}, \widetilde{\kappa}_{0}\right) \in V_{4}$ выполняем:

2.3.1. Полагаем $v_{\widetilde{\kappa}}:=0$. 
2.3.2. Для всех $(\widetilde{\tau}, \widetilde{\delta}) \in \Theta$ выполняем:

2.3.2.1. Если выполнено $\sim$ равенство $\widetilde{s}_{8}\left(\widetilde{\tau}+{ }_{16} \widetilde{\kappa}\right) \quad \oplus$ $\oplus \widetilde{s}_{8}\left(\widetilde{\tau}+{ }_{16} \widetilde{\kappa}+{ }_{16} 1\right)=\widetilde{\delta}$, то $v_{\widetilde{\kappa}}:=v_{\widetilde{\kappa}}+1$.

2.3.3. Полагаем

$$
\widetilde{K}^{[r]}:=\left\{\widetilde{\kappa} \in V_{4} \mid v_{\widetilde{\kappa}}=\max \left\{v_{\tilde{\lambda}} \mid \widetilde{\lambda} \in V_{4}\right\}\right\} .
$$

Выход: множество $\widetilde{K}^{[r]}$, величина $c_{0}$.

Если $c_{0}=1$, то переходим к этапу III.

Этап III. Нахождение бит $k_{0}^{(r)}, \ldots, k_{26}^{(r)}$ раундового ключа $\widehat{k}^{(r)}$.

Вход: $K^{[32]}, \ldots, K^{[r+1]}-$ множества кандидатов в раундовые ключи для раундов $32-(r+1)$.

1. Полагаем $\bar{k}:=\widehat{0}, \kappa:=\widehat{0}$.

2. Для всех $t \in\{0, \ldots, 26\}$ выполняем:

2.1. Полагаем $c:=0$.

2.2. Для всех $i \in\left\{1, \ldots, n_{0}^{(3.2 .2)}\right\}$ и для всех $\gamma_{3}=\left(\widetilde{\gamma}_{3,1}, \widetilde{\gamma}_{3,0}\right) \in V_{8}$ выполняем:

2.2.1. Полагаем

$$
\begin{gathered}
\alpha^{(0, i)}:=g_{\widehat{k}^{(1)}} \ldots g_{\widehat{k}^{(32-r)}}\left(\kappa \oplus \widehat{\varepsilon}_{26-t}, \alpha_{0}^{(32-r, i)}\right), \\
\alpha^{\prime(0, i)}:=g_{\widehat{k}^{\prime(1)}} \ldots g_{\widehat{k}^{(32-r)}}\left(\kappa \oplus \widehat{\varepsilon}_{26-t}, \alpha_{0}^{(32-r, i)} \oplus(\underbrace{0, \ldots, 0}_{21}, \gamma_{3}, 0,0,0)\right) .
\end{gathered}
$$

2.2.2. Вычисляем

$$
\alpha^{(32, i)}:=f_{k}\left(\alpha^{(0, i)}\right), \alpha^{(32, i)}:=f_{k^{\prime}}\left(\alpha^{(0, i)}\right) .
$$

2.2.3. Полагаем

$$
\begin{aligned}
\alpha^{(r, i)} & :=g_{\widehat{k}^{(r+1)}}^{-1}\left(\ldots g_{\widehat{k}^{(32)}}^{-1}\left(\alpha^{(32, i)}\right)\right), \\
\alpha^{\prime(r, i)} & :=g_{\widehat{k}^{\prime(r+1)}}^{-1}\left(\ldots g_{\widehat{k}^{\prime}(32)}^{-1}\left(\alpha^{\prime(32, i)}\right)\right) .
\end{aligned}
$$

2.2.4. Вычисляем

$$
\widehat{\lambda}=\left(\widetilde{\lambda}_{7}, \ldots, \widetilde{\lambda}_{0}\right):=\left(\widehat{\alpha}_{0}^{(r, i)} \oplus \widehat{\alpha}_{0}^{(r, i)}\right) \ggg 3 .
$$

2013. T. 4. № 2. C. 127-152 
2.2.5. Если $\widehat{\alpha}_{1}^{(r, i)} \oplus \widehat{\alpha}_{1}^{\prime(r, i)}=\widehat{0}$ и $\widetilde{\lambda}_{j}=\widetilde{0}, j=2, \ldots, 7$, то

2.2.5.1. Если $\widetilde{\gamma}_{3,1}=0$, то $c:=1, \widehat{\kappa}:=\widehat{\kappa} \oplus \widehat{\varepsilon}_{26-t}$, переходим к шагу III.2.1, $t:=t+1$ и опробуем следующее значение $\gamma_{3}$.

2.2.5.2. Если $\widetilde{\gamma}_{3,1} \neq 0$, то $c:=1, \bar{k}:=\bar{k} \oplus \widehat{\varepsilon}_{26-t}$, переходим к шагу III.2.1, $t:=t+1$ и опробуем следующее значение $\gamma_{3}$.

2.3. Если $c=0$, то завершаем работу алгоритма и возвращаем $(\varnothing, c)$.

3. Полагаем

$$
K^{[r]}:=\left\{\left(\widetilde{\kappa}, 0, \bar{k}_{26}, \ldots, \bar{k}_{0}\right) \cup\left(\widetilde{\kappa}, 1, \bar{k}_{26}, \ldots, \bar{k}_{0}\right) \mid \widetilde{\kappa} \in \widetilde{K}^{[r]}\right\}
$$

и завершаем работу алгоритма.

Выход: множество $K^{[r]}$ кандидатов в раундовые ключи $r$-го раунда, величина $c$. 